\title{
Correlation between maxillary sinus floor topography and relative root position of posterior teeth using Orthopantomograph and Digital Volumetric Tomography
}

\author{
Chaithra Kalkur ${ }^{1}$, Atul P Sattur ${ }^{2}$, Kruthika S Guttal ${ }^{3}$, Venkatesh G Naikmasur ${ }^{4}$, Krishna Burde ${ }^{5}$ \\ ${ }^{1}$ Senior Lecturer, Department of Oral Medicine and Radiology, Century International Institute of Dental Science and \\ Research Centre, Poinachi, Kasargod, Kerala, $2,4,5$ Professor, ${ }^{3}$ Associate Professor, Department of Oral Medicine and \\ Radiology, S.D.M College of Dental Sciences and Hospital, Sattur, Dharwad, Karnataka, India
}

\section{A B S T R A C T}

Background: Maxillary sinus and its close proximity to the oral cavity make it a common clinical concern for medical and dental practitioners. Due to anatomical complexities, it is difficult to evaluate the relation between the root apices of maxillary posterior teeth and the floor of maxillary sinus which is essential for diagnosis of sinus pathology, understanding the path of dental infection and planning of dental treatment. Aims and Objectives: To correlate the topographic relationship of the maxillary sinus floor to the maxillary posterior teeth roots as imaged by pairs of Orthopantomograph (OPG) and Digital Volumetric Tomography images (DVT). Materials and Methods: A total of 510 maxillary teeth from 85 patients were classified according to their topographic relationship to the maxillary sinus and measured according to their projection lengths on the sinus cavity using OPG and DVT modalities. Results: In cases of classification $0,85 \%$ and in classification $1,55.3 \%$ cases shows similar classification in both OPG and DVT. $28.5 \%$ of cases in both imaging modalities show classification 2 . Only $15.9 \%$ of teeth roots exhibits classification $3.11 .1 \%$ of cases showed classification 4 . The panoramic radiograph showed a statistically significant 2.24 times longer root projection on the sinus cavity in OPG comparison to DVT images. Conclusion: Teeth roots projecting in to the sinus in OPG, shows no vertical protrusion in to the sinus in DVT images. Hence DVT was better than OPG with measurements that were more exact and closer to anatomical reality.

\section{Access this article online}

Website:

http://nepjol.info/index.php/AJMS

DOI: $10.3126 /$ ajms.v8i1.15878

E-ISSN: 2091-0576

P-ISSN: 2467-9100

Key words: Maxillary sinus floor, Orthopantomograph, Digital Volumetric Tomography

\section{INTRODUCTION}

Cone Beam Computed Tomography (CBCT) is a new medical imaging technique which is based on a cone shaped $\mathrm{x}$ ray centered on a $2-\mathrm{D}$ detector that performs one rotation around the object, producing a series of 2-D images. These images are reconstructed in 3-D using a modification of the original cone-beam algoritham developed by Feldkamp et al in $1984 .{ }^{1}$ CBCT differs from medical CT Imaging in that the whole three-dimensional volume of data is acquired in the course of a single sweep of the scanner, using a simple,direct relationship between sensor and source. ${ }^{2}$ Synonyms for CBCT includes
Cone Beam Volumetric Tomography, Digital Volumetric Tomography, Dental Computed Tomography, Cone Beam Imaging and CB3D-Cone beam there -dimensional imaging. ${ }^{3,4}$

Digital Volumetric Tomography is a recent technology initially developed for angiography in 1982 and subsequently applied to maxillofacial imaging. DVT provides high definition, three dimensional digital data on precise anatomical information of all oral and maxillofacial structures at reduced cost and less radiation to patient, in comparison to traditional imaging systems like orthopantomography, which are limited by 
distortion, magnification changes, restricted clarity, lack of accuracy in measurements and not allowing for 3D modelling. ${ }^{5}$

Cone Beam scanners are based on a cone shaped beam of $\mathrm{x}$ - rays rotating around the object of interest giving a volume of data, using a 2-dimensional extended digital array as an area detector. The technique involves a single 360-degree scan in which the $\mathrm{x}$ - ray source and reciprocating area detector synchronously move around the patient's head, which is stabilized with a head holder. At certain degree intervals, single projection images, known as 'basis' images, are acquired. These are similar to lateral cephalometric images,each slightly offset from one another. This series of basis projection images is referred to as the projection data. ${ }^{6}$

Clinical applications for cone-beam imaging are,

1. Investigation of jaw pathology including cysts, tumors and fibro-osseous lesions.

2. Investigation of Pre-and post-implant assessment.

3. Orthodontic assessment, both dental development and skeletal base relationship.

4. Investigation of the bony components of the TMJ.

5. Assessment of wisdom teeth, in particular their relationship to the inferior dental canal and evaluation of facial trauma. ${ }^{7,8}$

The aims and objective of this study was to compare the imaging characteristics between panoramic radiographs and DVT of maxillary sinus floor to the posterior teeth roots and to correlate the topographic relationship of the maxillary sinus floor to the posterior teeth roots as imaged by pairs of panoramic radiographs and DVT images in a relatively large sample.

\section{MATERIALS AND METHODS}

A total of 85 patients were selected, out of which there were 49 males and 36 female patients. Their age ranged from 18 to 45 years. A thorough clinical examination was performed for these patients. Information regarding the nature \& purpose of study was thoroughly explained to every patient and a written consent was obtained from them. Patients with missing teeth, periapical pathology of maxillary posterior teeth and pregnant patient were excluded from the study.

All the patients were subjected for conventional (OPG) and digital imaging (DVT) evaluation. KODAK 9000C 3D Extra oral imaging system (Care stream Health, Inc. 150 Verona Street Rochester NY 14 608) was used for both obtaining OPG and DVT images.

\section{Exposure parameters}

- Panoramic radiography: 70-74 kVp, 14.3-15.1 mAs with scan time of 15.1 seconds.

- Digital Volumetric Tomography: 70 to $80 \mathrm{kVp}, 10$ $\times 10.8 \mathrm{mAs}$ with a scan time of 24 seconds.

The radiographic exposure for patients was well below the maximum permissible dose of $2.4 \mathrm{mSv}$ as per the NCRP guidelines. Radiation safety precautions such as filtration, collimation, and patient protection equipments like thyroid collar, lead apron and gonadal shield were used before subjecting the patients for imaging evaluation.

The correlation between maxillary sinus floor topography and related root position of posterior teeth was assessed by the following parameter, (Following classifications were taken from Sharan \& Madjar et al (2006)study).

Maxillary second premolars, the first and second molars, were taken for classification, because of the proximity of their root apices to the sinus floor. Only teeth whose root apices were clearly imaged in both radiographic techniques were included in the study. Inter examiner variation was determined by 2 observers at an interval of 1 week.

The topographic relationship of each root to the maxillary sinus floor in the panoramic radiograph and in the digital volumetric tomography were classified as,

0 - The root is not in contact with the cortical borders of the sinus;

1 - An inferiorly curving sinus floor, the root is in contact with the cortical borders of the sinus;

2 - An inferiorly curving sinus floor, the root is projecting laterally on the sinus cavity but its apex is outside the sinus boundaries;

3 - An inferiorly curving sinus floor, the root apex is projecting on the sinus cavity, and;

4 - A superiorly curving sinus floor enveloping part or allof the tooth root. ${ }^{9}$

OPG and DVT images were interpreted on HP L1910 19-inch square LCD Monitor with 1280 x 1024 screen resolution. In all the cases, the length of the apical part of the root intruded in to the floor of the sinus was measured in both radiographic techniques. However in $3 \mathrm{D}$ Volumetric imaging $1 \mathrm{~mm}$ tomographic sections in sagittal, axial and coronal planes were made. The measurements were taken from the root apex to the inferior wall of the sinus along the longitudinal axis of the root using Kodak Dental Imaging Software (Figures 1 and 2).

The results of the study were subjected to following statistical analysis using SPSS 18.0 software. Kappa 
Statistics was used to assess interobserver variation and to compare the classifications of the roots in the 2 imaging techniques. Paired $t$ test was used to analyse the mean difference between the root lengths superior to the sinus floor in the 2 imaging techniques. Correlation and Regression analyses were used to

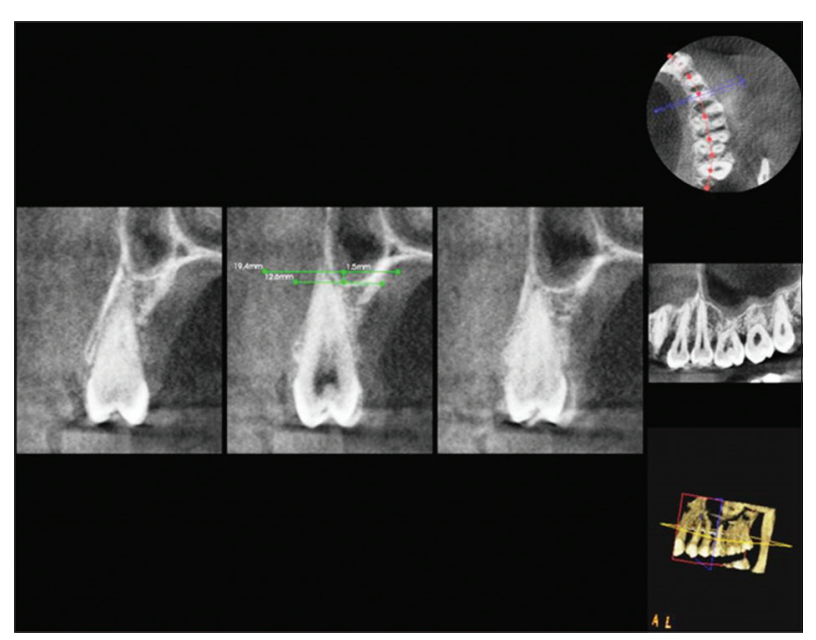

Figure 1: DVT showing the relation of maxillary left second pre molar with floor of the sinus in axial, coronal and sagittal sections and 3D reconstruction

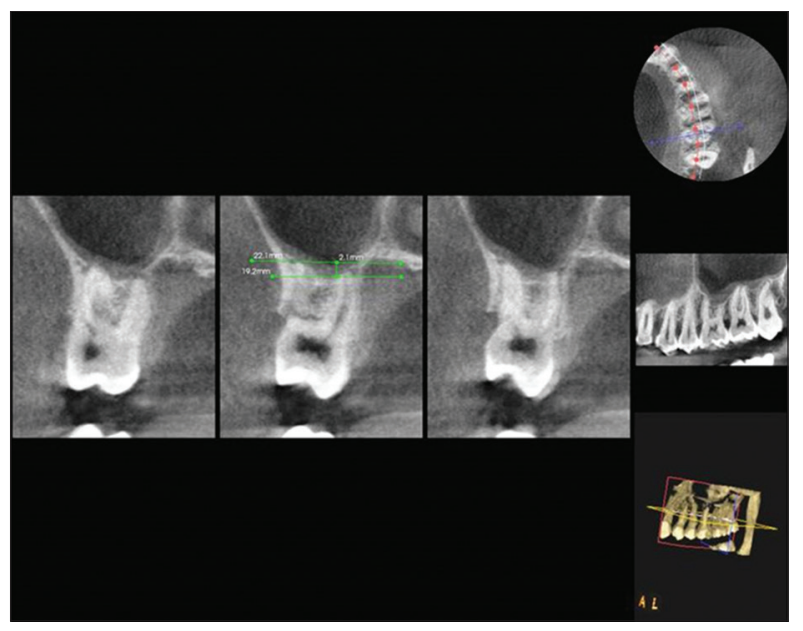

Figure 2: DVT showing the relation of maxillary left second molar with floor of the sinus in axial, coronal and sagittal sections and 3D reconstruction derive equations by comparing various lengths of projection of the roots superior to the sinus floor in the 2 imaging techniques.

\section{RESULTS}

The present study was conducted on 85 patients out of these, $49(57.6 \%)$ were males and $36(42.3 \%)$ were females. A total of 510 teeth (2010 roots) were assessed in these patients. Their age ranged from 18-45 years. Positional relationships between the floor of the maxillary sinus and maxillary second premolar, first and second molars were classified into 5 categories (Table 1).

In cases of classification $0,85 \%$ and in classification 1 , $55.3 \%$ cases show similar classification in both OPG and DVT. $28.5 \%$ of cases in both imaging modalities show classification 2 . Only $15.9 \%$ of teeth roots exhibits classification 3.11.1\% of cases showed classification 4. In cases in which DVT showed roots with classification 2 , most of the panoramic radiograph $(87 \%)$ showed classification 3. $88.2 \%$ of the cases that showed a root protruding into the sinus in the DVT (Classification 3) also showed root projection in the panoramic radiography (Table 2).

These were evaluated using Orthopantomography (OPG) and Digital Volumetric Tomography (DVT) images. Interexaminer variation was determined by 2 observers at an interval of 1 week. There was significant agreement between observer 1 and 2 suggestive of no interobserver variation $\left({ }^{*} \mathrm{p}<0.05\right)$ in any of the imaging modalities.

The mean difference between the root lengths superior to the sinus floor in the 2 imaging techniques by comparing OPG and DVT with respect to average grading of all teeth, the data showed that the root projection length in the panoramic radiograph was 2.24 times larger than the actual root protrusion length in the DVT. The difference was not statistically significant (Table 3).

\begin{tabular}{|c|c|c|c|c|c|c|c|}
\hline \multirow{2}{*}{$\begin{array}{l}\text { Type of } \\
\text { tooth }\end{array}$} & \multirow{2}{*}{$\begin{array}{l}\text { Type of } \\
\text { radiograph }\end{array}$} & \multicolumn{5}{|c|}{ Classification } & \multirow[t]{2}{*}{ Total } \\
\hline & & 0 & 1 & 2 & 3 & 4 & \\
\hline Second & OPG & 35 & 43 & 10 & 81 & 1 & 170 \\
\hline Premolar & DVT & 65 & 50 & 40 & 14 & 1 & 170 \\
\hline First & OPG & 10 & 40 & 3 & 113 & 4 & 170 \\
\hline Molar & DVT & 38 & 57 & 39 & 19 & 17 & 170 \\
\hline Second & OPG & 14 & 50 & 12 & 90 & 4 & 170 \\
\hline Molar & DVT & 30 & 82 & 20 & 19 & 19 & 170 \\
\hline Total & & 192 & 322 & 124 & 336 & 46 & 1020 (510 teeth ) \\
\hline
\end{tabular}




\begin{tabular}{|c|c|c|c|c|c|c|}
\hline \multirow[t]{2}{*}{ OPG } & \multicolumn{6}{|c|}{ DVT } \\
\hline & 0 & 1 & 2 & 3 & 4 & TOTAL \\
\hline 0 & 50 & 8 & 0 & 0 & 1 & $59(11.5 \%)$ \\
\hline 1 & 51 & 73 & 3 & 4 & 1 & $132(25.8 \%)$ \\
\hline 2 & 1 & 17 & 8 & 2 & 0 & $28(5.4 \%)$ \\
\hline 3 & 31 & 85 & $88(87 \%)$ & $45(88.2 \%)$ & 33 & $282(55.2 \%)$ \\
\hline 4 & 0 & 6 & 2 & 0 & 1 & $9(1.7 \%)$ \\
\hline Total & $133(26 \%)$ & $189(37 \%)$ & $101(19.8 \%)$ & $51(10 \%)$ & $36(7.05 \%)$ & 510 \\
\hline
\end{tabular}

Table 3: Comparison of OPG and DVT with respect to average gradings of all teeth by test

\begin{tabular}{lccccccc}
\hline Methods & Mean & Std.Dv. & Mean Diff. & SD Diff. & \% of difference & Paired $t$ & p-value \\
\hline OPG & 6.30 & 2.39 & & & & & \\
DVT & 4.06 & 2.27 & 2.24 & 1.68 & 35.48 & 12.2485 & $0.0000^{*}$ \\
\hline${ }^{*}<0.05$ & & & & & & &
\end{tabular}

\section{DISCUSSION}

The floor of maxillary sinus is formed by the lower third of the medial wall and the buccoalveolar wall of the maxilla. ${ }^{10}$ Paatero (1939) confirmed this finding and according to his study order of frequency of the teeth, in close proximity with the sinus are, second molar, the first molar, third molar, second premolar, first premolar and canine. ${ }^{11}$ Stafne (1985) estimated that $15-75 \%$ of the time, sinusitis occurs through a dental cause although the true incidence is difficult to determine accurately. ${ }^{10}$

Ingle (1965) believed that contact between the maxillary sinus floor and inflammatory lesions resulted in the development of chronic sinusitis. It is also accepted that symptoms of maxillary sinusitis can emulate pain of dental origin, and a careful differential diagnosis is essential when dealing with pain in the maxillary posterior area. $^{12}$

Freisfeld et al were the first to suggest a classification of the relationship between the teeth and the sinus, but their method of classification was applicable only to first molars, but did not relate to the sinus floor topography, and did not include a situation of a root that projects laterally on the sinus. ${ }^{\text {? }}$

Kwak et al suggested a more elaborate classification proposed by Freisfeld et al, but it was only applicable to CT images. They suggested 5 vertical relationships and 3 horizontal relationships in CT images only, finding that the most frequent vertical relationship was a sinus floor that was not contacting the tooth roots. It relates to the convexity or concavity of the sinus floor in the area of the teeth roots and is applicable to all posterior maxillary teeth and to both panoramic radiography and CT. ${ }^{9}$
Nimigean et al reported that the maxillary height in the region of the first premolar and first molar was significantly higher in men than in women, and the most lower zone of maxillary sinus floor at the level of 2 nd molar, at $50 \%$ in men and $56 \%$ in women. ${ }^{13}$

Ohba T et al radiologically compared the depth of sinus floor, but did not observe any statistical difference between the right and left sides. ${ }^{13}$ Kilica et al conducted a study on 92 Patients using Dental CBCT. No statistically significant differences were found between the measurements for right and left sides $(\mathrm{P}>.05)$ or between female and male patients $(\mathrm{P}>.05) .{ }^{14}$

Present study shows in cases of roots not contacting the sinus floor (classification 0), or contacting but not projecting on the sinus cavity (classification1), 85\% and $53 \%$ cases shows similar classification in both OPG and DVT. In these cases OPG is sufficient to provide the clinician the true relationship between the maxillary sinus and posterior teeth roots. $28.5 \%$ of cases in both imaging modalities show classification 2, suggesting the positional relationship of sinus floor, either anterior or posterior to the second premolar, first and second molar.

Only $15.9 \%$ of teeth roots projecting on the sinus cavity in the panoramic radiograph were protruding vertically into the sinus in the DVT images. Thus, a 3-dimensional image is required in these cases in order to ascertain the true relationship between the sinus and the tooth root. $11.1 \%$ of cases showed similar classification in case of superiorly curving sinus floor in DVT may be achieved by orthogonal, curved and oblique slicing compare to the single cross sections of tooth in the CT.

In overall comparison of maxillary teeth roots in relation to the floor of sinus using OPG and DVT. In our study, 
cases in which DVT showed roots with classification 2, $87 \%$ showed classification 3 in OPG. However in $88.2 \%$ of the cases that showed a root protruding into the sinus in DVT, (class 3) also showed root projection in the OPG.

This could be due to 2-dimensionality of the panoramic radiograph, which causes roots that are buccal/linqual to the sinus to be projected on the sinus cavity. This can be compared with Sharan \& Madjar et al study. Here $86 \%$ of the roots with classification 2 in the CT were projecting on the sinus cavity in the panoramic radiograph, and $91 \%$ of the cases showed a root protruding vertically into the sinus in the CT also showed root projection on the sinus in the panoramic radiograph.

Therefore, when presented with a panoramic radiograph alone, the clinician cannot determine whether the root is actually protruding into the sinus or not. This is in contrast to the cross sectional imaging of the DVT, which allows an accurate interpretation of the true buccolingual relationships of the teeth roots to the sinus. Thus, panoramic radiograph presents certain drawbacks, such as superimposition of anatomic structures, horizontal and vertical magnification and a lack of cross-sectional information. Formation of ghost images on the side opposite the object, mesiodistal and vertical enlargement, blurring of some structures, spreading of a curved structure over a plane.

The average the root projection length in the panoramic radiograph was 2.24 times larger than the actual root protrusion length in the DVT with no significant differences between root types. This result is again explained by the 2-dimensionality of the panoramic radiograph, and it occurs when sinus recesses penetrate into the interradicular area. In these cases, the panoramic radiograph will show a significant part of the root projecting on the sinus, but the DVT will show that only the apical part of the root penetrates the sinus and the rest is medial/lateral to it. The large difference between the 2 imaging techniques cannot be explained simply by the vertical magnification of the panoramic image because it is only $1.27 \%$.

Arbel Sharan et al reported that the panoramic radiographs showed a statistically significant 2.1 times longer root projection on the sinus cavity in comparison to the root protrusion length into the sinus measured by using CT images.

In our study following formula was derived to predict the root protrusion length in the DVT from the root projection length in the panoramic radiograph:
Root protrusion length in DVT $=-0.3588+0.7021$ (Root projection length in OPG)

This formula is useful in correlating the relationship of maxillary sinus floor to the posterior teeth roots in both OPG and DVT. Hence it is used in the field of Implantology, endodontics, in case oroantral communications and pathology involving maxillary sinus.

\section{CONCLUSION}

It is difficult to analyse precisely a three dimensional sinus with a one dimensional Panoramic radiograph. The clinician cannot determine whether the root is actually protruding into the sinus or not. This is in contrast to the cross-sectional image of the DVT, which allows an accurate interpretation of the true buccolingual relationships of the teeth roots to the sinus. Any part of the root that is superior to the sinus floor in the DVT should be protruding into the sinus. DVT was better than panoramic radiograph with measurements that were more exact and closer to anatomical reality.

\section{ACKNOWLEDGEMENT}

The authors are grateful to the Department of Oral Medicine \& Radiology, S D M College of Dental Sciences and Hospital, Sattur, Dharwad, Karnataka.

\section{REFERENCES}

1. Patel S, Dawood A, Pitt Ford T and Whaites E. The potential applications of cone beam computed tomography in the management of endodontic problems. International Endodontic Journal 2007; 40:818-830.

2. Nakajimaa A, Sameshimab GT, Araic $Y$, Hommed $Y$, Shimizue $N$ and Dougherty $\mathrm{H}$. Two- and Three-dimensional Orthodontic Imaging Using Limited Cone Beam-Computed Tomography. Angle Orthod 2005; 75:895-903.

3. Scarfe WC and Farman AG. Cone Beam Computed Tomography. Oral Radiology, Principles and Interpretation.6th ed. Noida, India: Elsevier, 2009; 225-243.

4. Molteni R. The so-called cone beam computed tomography technology. Dentomaxillofac Radiology 2008; 37:477-478.

5. Raichur PS, Setty SB, Thakur SL and Naikmasur VG. Comparison of Radiovisiography and Digital volume tomography to direct surgical measurements in the detection of infrabony defects. $J$ Clin Exp Dent 2012; 4(1):43-47.

6. Ahmad M and Freymiller E. Cone Beam Computed Tomography: Evaluation of Maxillofacial Pathology. J Calif Dent Assoc 2010:38(1):41-47.

7. Nakajimaa A, Sameshimab GT, Araic Y, Hommed $Y$, Shimizue $\mathrm{N}$ and Dougherty $\mathrm{H}$. Two- and Three-dimensional Orthodontic Imaging Using Limited Cone Beam-Computed Tomography. Angle Orthod 2005; 75:895-903.

8. Scarfe WC and Farman AG. What is Cone-Beam CT and How Does it Work?. Dent Clin N Am 2008; 52: 707-730. 
9. Sharan A, Madjar D and Hashomer T. Correlation between maxillary sinus floor topography and related root position of posterior teeth using panoramic and cross-sectional computed tomography imaging. Oral Surg Oral Med Oral Pathol Oral Radiol Endod 2006; 102:375-381.

10. Malik NA. Textbook of Oral and Maxillofacial Surgery. New Delhi: Jaypee, 2005; 525-526.

11. Scarfe WC and Farman AG. Cone Beam Computed Tomography. Oral Radiology, Principles and Interpretation.Noida, India: Elsevier, 2009; 225-243.
12. Hauman $\mathrm{CH}, \mathrm{Chandler} \mathrm{NP}$ and Tong DC. Endodontic implications of the maxillary sinus: a review. Int Endod J 2002; 35:127-141.

13. Nimigean V, Nimigean VR, Măru N, Sălăvăstru DI, Bădiţ D and Tuculină MJ. The maxillary sinus floor in the oral implantology. Romanian Journal of Morphology and Embryology 2008; 49(4):485-489.

14. Kilica C, Kamburoglub K, Yukselc SP and Ozend T. An Assessment of the Relationship between the Maxillary Sinus Floor and the Maxillary Posterior Teeth Root Tips using Dental Cone-beam Computerized Tomography. Eur J Dent 2010; 4:462-467.

\section{Authors Contribution:}

CK and APS - Concept and design of the study, collected data, Data Acquisition, Data Analysis, Manuscript preparation, Drafting and Revision of manuscript, Editing and Final Approval; KSG - Data Analysis, Revision of Manuscript, reviewed the literature, Editing and Final Approval; VGN - Concept and design of the study, Editing and Final Approval; KB - Critical revision of the manuscript and Editing and Final Approval.

Source of Support: Nil, Conflict of Interest: None declared. 